\title{
Exchange coupled domain walls: Resonance in multilayers
}

R. L. Stamps, P. E. Wigen, and A. S. Carriço

Citation: Journal of Applied Physics 81, 5370 (1997);

View online: https://doi.org/10.1063/1.364600

View Table of Contents: http://aip.scitation.org/toc/jap/81/8

Published by the American Institute of Physics

\section{Articles you may be interested in}

Asymmetric spin-wave dispersion due to Dzyaloshinskii-Moriya interaction in an ultrathin $\mathrm{Pt} / \mathrm{CoFeB}$ film Applied Physics Letters 106, 052403 (2015); 10.1063/1.4907173

Spin-wave propagation in a microstructured magnonic crystal Applied Physics Letters 95, 262508 (2009); 10.1063/1.3279138

Theory of long-wavelength spin waves in exchange biased bilayers Journal of Applied Physics 89, 7651 (2001); 10.1063/1.1357867

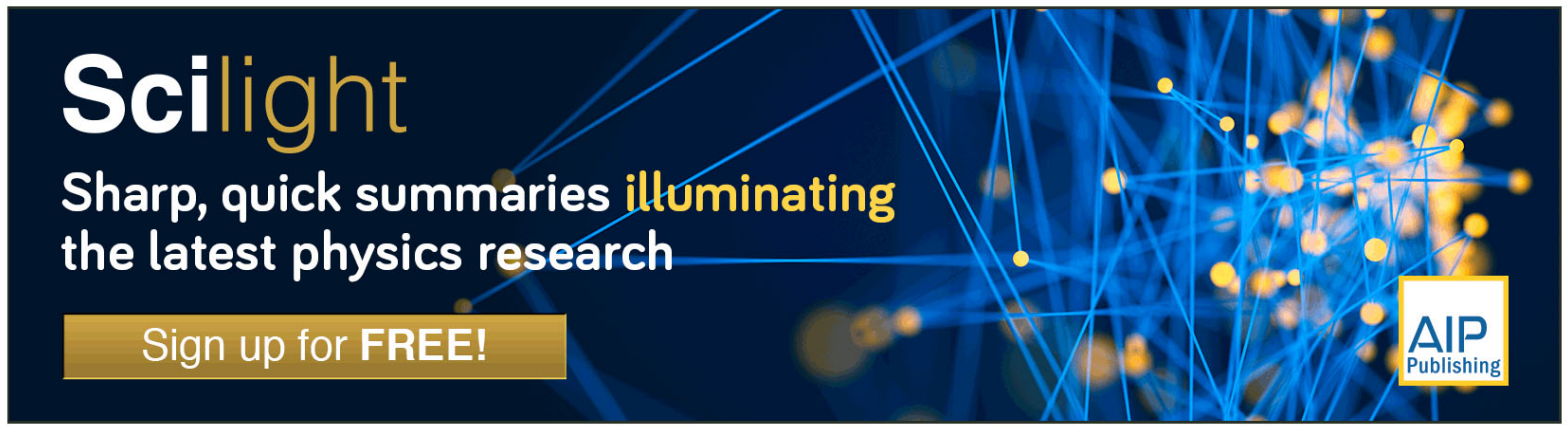




\title{
Exchange coupled domain walls: Resonance in multilayers
}

\author{
R. L. Stamps and P. E. Wigen \\ Department of Physics, Ohio State University, Columbus, Ohio 43210
}

\author{
A. S. Carriço \\ Departamento de Fisica, Centro de Ciencias Exatas, Universidade Federal do Rio Grande do Norte, \\ 59072-970 Natal/RN, Brazil
}

Exchange coupling between magnetic films can lead to an attractive force between domain walls in the separate films. The coupling between the films allows for small amplitude oscillations around the equilibrium configuration of the walls, analogous to optic and acoustic type spin wave resonance modes. Since the restoring force acts only over the length of a domain wall, this suggests the possibility of sensitive measurements of the local coupling by studying domain wall resonances. The effects of applied fields are also examined. With antiferromagnetic coupling, small static fields push the walls apart and result in different behaviors of the acoustic and optic domain wall resonances. (C) 1997 American Institute of Physics. [S0021-8979(97)50508-1]

Studies of domain configurations and domain walls in magnetic multilayers have provided valuable insights into the effects and character of interactions within and between magnetic layers. ${ }^{1}$ An interesting feature of multilayers are correlations between domain walls in separated films. ${ }^{2}$ Oscillations of domain wall pairs in single thin films have been predicted for certain wall configurations. ${ }^{3,4}$ In this article we consider a new kind of restoring force responsible for wall oscillations-interlayer exchange coupling between magnetic films.

To date, domain walls in multilayers have only been studied in terms of their static properties. Exchange coupling between domain walls on adjacent films can, however, lead to interesting dynamic effects. Consider two antiparallel coupled thin ferromagnetic films. The coupling energy will be smallest if walls in each film are positioned directly beneath one another as shown in Fig. 1(a) for two Neél walls. In this figure the arrows represent the local orientation of the magnetization in each film. Small deviations from this configuration, depicted in Fig. 1(b), increase the energy of the

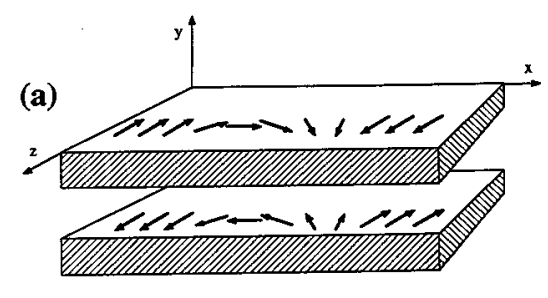

(b)

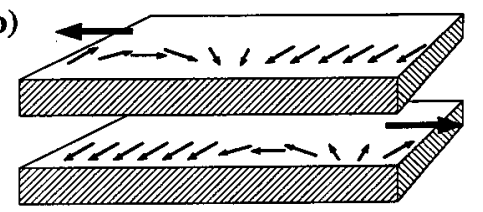

FIG. 1. Schematic illustration of relative motion and orientation of the magnetizations for Neél walls in two antiparallel coupled films. In (a) the equilibrium configuration is shown and in (b) the walls are displaced a small amount with a corresponding increase in energy due to the coupling. structure and result in torques on the spins in the walls of each film. Harmonic oscillations are then possible with a natural frequency that depends on the magnitude and sign of the interfilm coupling.

Domain wall resonance differs from spin wave resonance in that the precession of spins in the domain walls results in a translation of the domain wall along the film. Wall resonance frequencies are therefore determined by inertia involved in the translation and the nature of restoring "forces" incurred by the relative motion of the walls. We describe below a theory for the frequencies of oscillations when the restoring force is due to interfilm coupling.

We consider two exchange coupled ferromagnetic films. The films have identical magnetic properties and are assumed to be very thin so that Neél type walls are preferred. The wall profile is assumed to depend on position in only one direction. At equilibrium the spins lie in the film planes. Angles $\theta$ and $\phi$ are used to specify the orientation of the magnetization as a function of position $x$ in each film. The magnetization in the separate films is labeled $\mathbf{m}_{1}$ and $\mathbf{m}_{2}$.

The energy for the uncoupled wall system is assumed to have a usual form for one-dimensional walls ${ }^{5}$ and includes a uniaxial anisotropy, intrafilm exchange, applied static field $h_{s}$, and a demagnetizing factor for the thin film geometry. Our problem differs by the inclusion of an interfilm coupling $J$,

$$
\begin{aligned}
E_{\mathrm{ex}}= & J \int\left(\sin \theta_{1} \cos \phi_{1} \sin \theta_{2} \cos \phi_{2}\right. \\
& \left.+\sin \theta_{1} \sin \phi_{1} \sin \theta_{2} \sin \phi_{2}+\cos \theta_{1} \cos \theta_{2}\right) d x
\end{aligned}
$$

The subscripts 1 and 2 identify the film.

Interfilm and intrafilm magnetostatic are assumed small in comparison to other energies in the problem and are neglected for simplicity. Approximate solutions for the profiles specified by $\theta_{1}, \theta_{2}, \phi_{1}$, and $\phi_{2}$ are found by using a variational method involving trial solutions for the coupled equations. The trial solutions describe independent walls in uncoupled films. For antiparallel coupling these are given by

$$
\theta_{1}=\cos ^{-1}\{\tanh [(x) / \Delta]\} \quad \text { and } \quad \phi_{1}=0,
$$




$$
\theta_{2}=-\cos ^{-1}\{-\tanh [(x) / \Delta]\} \quad \text { and } \quad \phi_{2}=\pi .
$$

The wall width $\Delta$ is used as a variational parameter and the energy of the static coupled wall structure is found by substituting the solutions from Eqs. (2) and (3) into the wall energy and minimizing with respect to $\Delta$. Corresponding expressions for parallel walls are used in the case of parallel coupling.

Wall motion involves translations of $\theta_{1}$ and $\theta_{2}$. This perturbs the wall profile by an amount $\delta x(d \theta / d x)$ to first order where $\delta x$ is a small translation of the wall. Translation of the walls also involve fluctuations out of the film plane. These are given by $\psi_{1}$ and $\psi_{2}$ representing deviations of $\phi_{1}$ and $\phi_{2}$ from their equilibrium values. We expand the wall energy to second order in the translation variables $x_{1}, x_{2}$ and the outof-plane fluctuations $\psi_{1}$, and $\psi_{2}$, and evaluate the resulting integrals using the trial wall profile solutions.

A key feature is the form of the interfilm coupling term. After expanding the energy to second order in $x_{1}$ and $x_{2}$, the coupling has the form $J\left(x_{1}-x_{2}\right)^{2}$, which leads to the interfilm exchange restoring force on the walls. We note that this is intrinsic to the interfilm coupling and does not involve any defects or pinning centers.

The validity of this approach depends on the magnitude of $\delta x$ and the strength of $J$. When $\delta x=0$, the trial solutions are identically the uncoupled wall solutions regardless of the strength of $J$. Interfilm exchange energy is only involved when relative motions of the walls lead to deviations from perfect antiparallel alignment of the spins in the separate films. When the energy of these deviations from antiparallel alignment are large, then the walls can be strongly deformed and the variational procedure will fail. This is discussed in relation to a numerical check on the validity of the approach in Ref. 6. In the following we restrict our calculations to cases where the $\delta x$ are much smaller than a domain wall width.

Equations of motion can be constructed using the methods of Ref. 5. These have the form $(2 M / \gamma) d x_{i} / d t=d \sigma / d \psi_{i}$ and $(2 M / \gamma) d \psi_{i} / d t=-d \sigma / d x_{i}$, where $\gamma$ is the gyromagnetic ratio. Resonance frequencies $\omega$ can then be determined by assuming time varying solutions of the form $\exp (-i \omega t)$ for $x_{1}, x_{2}, \psi_{1}$, and $\psi_{2}$.

One nonzero mode exists for antiparallel coupling with the frequency

$$
\left(\omega_{o} / \gamma\right)^{2}=4 \pi M H_{e},
$$

where $H_{e}=2 J / M$. The subscript $o$ denotes optic meaning that the separate walls oscillate out of phase with one another. An in-phase acoustic mode can exist only if there are other restoring forces present. For example, the inclusion of a phenomenological effective restoring field $H_{K}$ (a simplified representation of pinning by a defect, for example) gives an acoustic mode frequency for the antiparallel case of

$$
\left(\omega_{a} / \gamma\right)^{2}=\left(H_{e}+4 \pi M\right) H_{K} .
$$

The optic mode is also modified by the presence of $H_{K} \cdot{ }^{6}$ When the interfilm coupling is zero, both frequencies reduce to the single uncoupled wall resonance frequency.

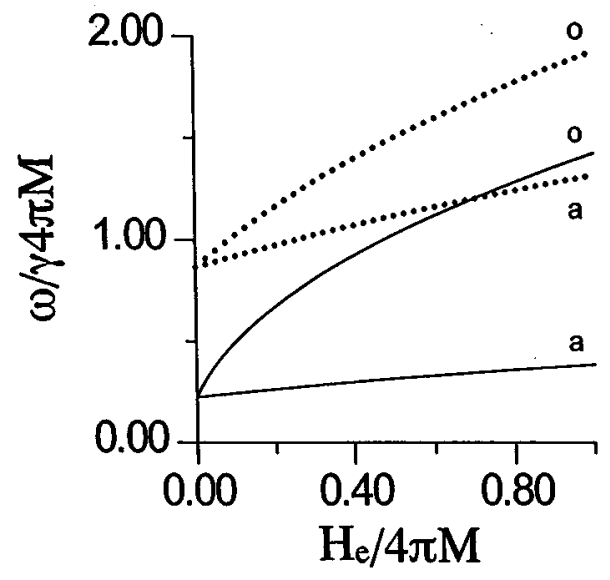

FIG. 2. Frequencies of the spin wave and domain wall resonances for the antiparallel coupled configurations as functions of coupling strength $H_{e}$. The solid lines are acoustic $a$ and optic $o$ domain wall resonances and the dotted lines are the acoustic and optic spin wave resonances.

The frequencies for domain wall resonance with antiparallel coupling are shown in Fig. 2 as functions of $H_{e}$. The results are given in unitless frequency and field variables defined by $\omega / \gamma 4 \pi M$ and $H_{e} / 4 \pi M$. In all cases $H_{K} / 4 \pi M=0.05$ which allows for a nonzero acoustic mode. Spin wave resonance frequencies are also shown for comparison. ${ }^{7,8}$ The difference in spin wave resonance and wall resonance frequencies is due mostly to the in-plane uniaxial anisotropy $K$. Note that a large interfilm exchange can cause the optic wall resonance mode to have a frequency greater than the acoustic spin wave branch.

The motion of the walls can be thought of as similar to two masses connected by a spring. Acoustic- and optic-type oscillations are possible with an effective mass for each wall determined by interfilm coupling and magnetostatic energies. The oscillation frequencies follow directly as a ratio of the restoring force to the effective mass. Because magnetic anisotropy determines the wall profile, and walls are able to move without changing shape, a result is that the frequencies for wall resonance do not contain the anisotropy gap of spin wave resonance and are usually much smaller than spin wave frequencies. We note that the effective masses are different for the two modes. In the present case, the acoustic mode effective mass contains contributions from interlayer magnetic coupling whereas the optic mode effective mass depends only on $M$.

We now consider the case of a small static applied field. The presence of a static applied field creates pressure on the walls and causes them to move apart in the case of antiparallel interfilm coupling. The equilibrium positions for the walls are found by minimizing the wall energy with respect to the position variables $x_{1}, x_{2}, \Psi_{1}$, and $\Psi_{2}$ with a nonzero applied field. Calculation of the resonance frequencies then procede along the lines describe above.

Wall resonance frequencies in the antiparallel configuration are shown in Fig. 3 as a function of field $h_{s}$. The field is small in order that the walls are not pushed too far apart and so overlap. The dotted lines are the acoustic modes $\omega_{a}$ and the solid lines are optic modes $\omega_{o}$. Coupling parameters 


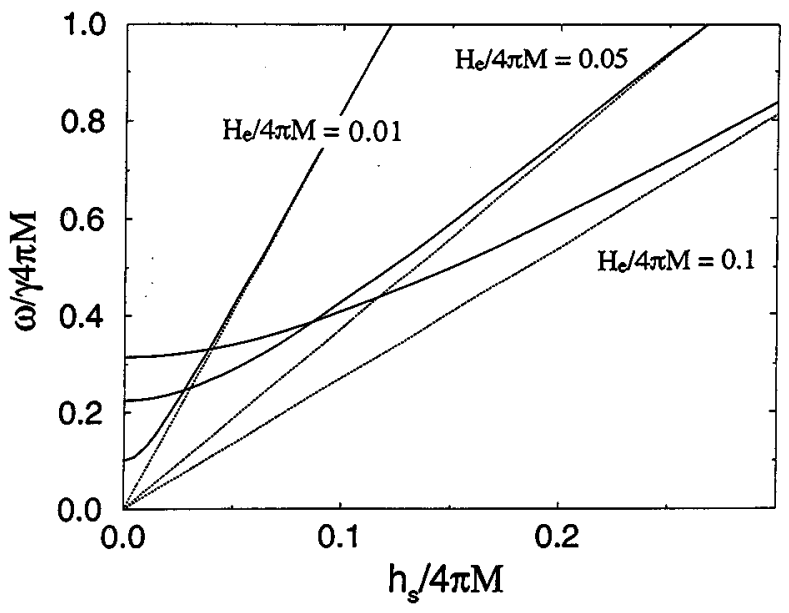

FIG. 3. Frequencies for antiparallel coupling as a function of applied field. The static applied field $h_{s}$ pushes the walls apart and the frequencies represent small oscillations about the equilibrium position. The acoustic modes (dotted lines) increase linearly with field in contrast to the optic modes.

$H_{e} / 4 \pi M$ between 0 and 0.1 are used. In this example $H_{K}=0$ so the only restoring force is due to $H_{e}$.

The striking feature is the existence of the acoustic mode for a small applied field. In the absence of an external applied field, the acoustic mode has zero frequency since it takes no energy for the walls to translate equal amounts in the same direction. In the antiparallel configuration with a nonzero applied field, acoustic mode motion always costs Zeeman energy for translation of one of the walls. Translation of the other wall in the acoustic mode gains Zeeman energy. This means that the two walls experience unequal forces with the result of a nonzero frequency for the acoustic mode. The field dependence of the optic mode, however, appears because of higher-order deformations of the domain wall width. ${ }^{6}$

In conclusion, we note that since the domain wall resonance is due to restoring forces localized to the region of the domain wall, the frequencies of the resonances are determined by coupling across areas with dimensions determined by the domain wall widths. These lengths are on the order of $100 \AA$ in high-anisotropy ferromagnetic metals. Observation of domain wall resonances would therefore allow for investigations of coupling mechanisms on a much smaller length scale than possible with ferromagnetic resonance or Brillouin light scattering. These measurement techniques provide values for the interfilm coupling averaged over lengths determined by the wavelength of the probing microwave or optical field, which puts the length scales at $1000 \AA$ lengths and more.

Work by R.L.S. and A.S.C. was supported in part by the CNPq. R.L.S. also thanks the UFRN for support.

${ }^{1}$ M. Rührig, R. Schäfer, A. Hubert, R. Mosler, J. A. Wolf, S. Demokritov, and P. Grünberg, Phys. Status Solidi A 124, 635 (1991).

${ }^{2}$ L. J. Heyderman, H. Niedoba, H. O. Gupta, and I. B. Puchalska, J. Magn. Magn. Mater. 96, 125 (1991).

${ }^{3}$ J. C. Slonczewski, J. Appl. Phys. 55, 2536 (1984).

${ }^{4} \mathrm{H}$. Braun and O. Brodbeck, Phys. Rev. Lett. 70, 3335 (1993).

${ }^{5}$ A. P. Malozemoff and J. C. Slonczewski, in Applied Solid State Science, edited by R. Wolfe (Academic, London, 1979).

${ }^{6}$ R. L. Stamps, P. E. Wigen, and A. S. Carrico, Phys. Rev. B (to be published).

${ }^{7}$ B. Heinrich, Z. Celinski, J. F. Cochran, A. S. Arrott, K. Myrtle, and S. T. Purcell, Phys. Rev. B 47, 5077 (1993).

${ }^{8}$ R. L. Stamps, Phys. Rev. B 49, 339 (1994). 